\title{
The internal structure of ZZ Cet stars using quantitative asteroseismology: The case of R548
}

\author{
N. Giammichele ${ }^{1}$, G. Fontaine ${ }^{1}$, P. Brassard ${ }^{1}$, and S. Charpinet ${ }^{2}$ \\ ${ }^{1}$ Département de physique, Université de Montréal, \\ C.P. 6128, Succursale Centre-Ville, Montréal, QC H3C3J7, Canada \\ email: noemi@astro.umontreal.ca \\ ${ }^{2}$ Université de Toulouse, UPS-OMP, IRAP, Toulouse, France; \\ CNRS, IRAP, 14 avenue Edouard Belin, 31400, Toulouse, France
}

\begin{abstract}
We explore quantitatively the low but sufficient sensitivity of oscillation modes to probe both the core composition and the details of the chemical stratification of pulsating white dwarfs. Until recently, applications of asteroseismic methods to pulsating white dwarfs have been far and few, and have generally suffered from an insufficient exploration of parameter space. To remedy this situation, we apply to white dwarfs the same double-optimization technique that has been used quite successfully in the context of pulsating hot B subdwarfs. Based on the frequency spectrum of the pulsating white dwarf R548, we are able to unravel in a robust way the unique onion-like stratification and the chemical composition of the star. Independent confirmations from both spectroscopic analyses and detailed evolutionary calculations including diffusion provide crucial consistency checks and add to the credibility of the inferred seismic model. More importantly, these results boost our confidence in the reliability of the forward method for sounding white dwarf internal structure with asteroseismology.
\end{abstract}

Keywords. stars: oscillations, white dwarfs

\section{Introduction}

Asteroseismology is a unique tool that allows us to probe the internal structure of a pulsating star. Until recently however, applications of the method to pulsating white dwarfs were few, and greatly suffered from an insufficient exploration of parameter space. The use of fully evolutionary models tend to propagate errors, and by the end of the calculation, uncertainties are greatly boosted. Moreover, such calculations are lenghty and it is impossible to thoroughly explore parameter space. To remedy this situation, we have begun to apply to white dwarfs the same double-optimization technique based on the forward approach that has been successfully used in the context of pulsating hot B subdwarfs (see, e.g., the review of Charpinet et al. 2009a). Targeted white dwarfs were chosen lying close to the blue edge, where the simplest pulsators are found. Even if only a few modes are excited in such stars, they still contain individually quite a bit of complementary information since they are low-order modes, far away from the asymptotic regime. We took advantage of a previously unexploited data set of very high $\mathrm{S} / \mathrm{N}$, obtained with the CFHT/LAPOUNE combination to extract pulsation periods in the first part of this venture (Giammichele et al. 2013a). We carried out our seismic analysis on the basis of five very certain periods observed in R548 (an improved extraction exercise was performed since Giammichele et al. 2013a and slightly different pulsation periods were obtained and used hereinafter). Results are presented in Giammichele et al. (2013b). This work contains the final validations of this asteroseismic exercise. 


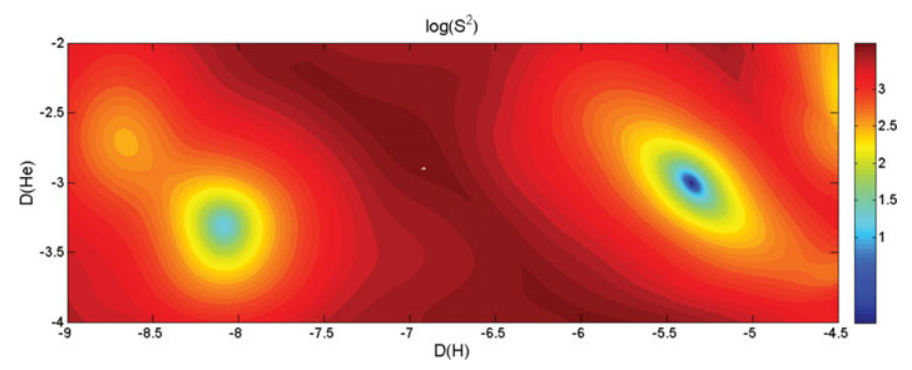

Figure 1. Contour map of the merit function as a function of the top of the transition zones $D(\mathrm{H})$ and $D(\mathrm{He})$, while the remaining parameters are fixed at their optimized values. $D(\mathrm{H})$ and $D(\mathrm{He})$, natural parameters of the optimization exercise, are directly related to the more familiar parameters $\log \left(M_{\mathrm{H}} / M_{*}\right)$ and $\log \left(M_{\mathrm{He}} / M_{*}\right)$ through: $\log \left(M_{\mathrm{H}} / M_{*}\right)=D(\mathrm{H})+C$ (similar for the helium layer) where $C$ is a small positive term, slightly dependent on the model parameters, determined by the mass of hydrogen or helium present inside the transition zones.

\section{Internal structure}

\subsection{Optimization procedure}

Given the high quality of our data, our goal is now to apply the same forward approach that has been successfully performed on hot pulsating subdwarfs. The technique relies on a first optimization leading to the best possible fit between the five observed periods (in the present case) with five periods out of the theoretical spectrum of a given stellar model. The second optimization step next leads to the best model in parameter space. To fully define a static white dwarf model, we have to specify the surface gravity (alternatively, the mass), the effective temperature, the chemical stratification in the envelope, and the core composition. Since low radial-order modes are quite insensitive to the prescribed convective efficiency, we fixed the latter to the so-called ML2 $/ \alpha=1.0$ prescription and did not vary it in parameter space. The quality of the match is then quantified through a second optimization by a merit function $S^{2}$ (an unweighted $\chi^{2}$ ), which is minimized as a function of $T_{\text {eff }}, \log g, \log \left(M_{\mathrm{H}} / M_{*}\right)$, and $\log \left(M_{\mathrm{He}} / M_{*}\right)$, given a core composition. Thus, for every set of $T_{\text {eff }}, \log g, \log \left(M_{\mathrm{H}} / M_{*}\right)$, and $\log \left(M_{\mathrm{He}} / M_{*}\right)$, we get a unique period spectrum to compare with the observed frequencies.

The forward procedure as implemented in Montréal (a full grid approach) necessitates the computation of many millions of models and their respective period spectra in a reasonable amount of time, and the use of our dedicated cluster of 320 nodes is a necessity. The same exercise with fully evolutionary sequences, including diffusion would be impracticable. It is worth repeating that there is no a priori guarantee to find an optimal model and, moreover, to find an optimal model that would be consistent with independent spectroscopic determinations of the atmospheric parameters.

The resulting optimum asteroseismic model presents a very low $S^{2}=0.83$, with $T_{\text {eff }}=12095 \mathrm{~K}, \log g=8.070, \log \left(M_{\mathrm{H}} / M_{*}\right)=-4.54$, and $\log \left(M_{\mathrm{He}} / M_{*}\right)=-2.42$. Details on the mode identification, given automatically by the solution, are presented in Table 1. Consecutive values of the radial order $k$, are perfectly consistent with the prediction from nonadiabatic theory that pulsation modes are excited in consecutive modes. Moreover, the nice agreement between the newest spectroscopic determination of $T_{\text {eff }}=11980 \mathrm{~K}$, $\log g=8.06$ (kindly provided to us by P. Bergeron) and our seismic determination of atmospheric parameters boosts our confidence in the validity of the solution found.

\subsection{Chemical stratification}

Figure 1 unambiguously suggests that R548 is composed of a thick hydrogen and thick helium layer as indicated by the deeper minimum. A much less convincing "thin" envelope 
Table 1. Mode identification for the best-fitting model with a mixed C-O core composition (mass fraction: 60-40).

\begin{tabular}{cccc}
\hline Observed periods & Fitted periods & $l$ & $k$ \\
\hline 212.96 & 212.98 & 1 & 2 \\
274.53 & 274.60 & 1 & 3 \\
318.43 & 318.18 & 1 & 4 \\
334.12 & 334.96 & 1 & 5 \\
186.89 & 187.14 & 2 & 4 \\
\hline
\end{tabular}

possibility also appears in the figure, but can readily be ruled out on the grounds of its significantly degraded merit function. There is no degeneracy in the optimized grid of seismic solutions. The chemical stratification with thick hydrogen and helium layers found for R548 ought to be reliable.

A first validation of the use of the forward method in this context is illustrated in Figure 2. We explore the importance of the imposed steepness of the chemical profile at the interface between the $\mathrm{H} / \mathrm{He}$ and $\mathrm{He} / \mathrm{C}-\mathrm{O}$ layers in our static models. While the transition between the $\mathrm{H} / \mathrm{He}$ layers leaves a stronger signature on the period spectrum than the deeper $\mathrm{He} / \mathrm{C}-\mathrm{O}$ interface, we still find a very narrow and well-defined minimum for $a(\mathrm{H} / \mathrm{He})=1.3$ and $a(\mathrm{He} / \mathrm{C}-\mathrm{O})=3.3$, the parameters defining steepness. Here again, these two parameters do not lead to any degeneracy in the asteroseismic solution. More importantly, evolutionary calculations, including diffusion, confirm transition steepness values of $a(\mathrm{H} / \mathrm{He})=1.31$ and $a(\mathrm{He} / \mathrm{C}-\mathrm{O})=3.20$, in almost perfect agreement with our asteroseismic values.

\subsection{Core composition}

The distribution of the Brunt-Väisälä frequency in the deepest layers is slightly but sufficiently perturbed by the different possible core compositions to allow us to probe the bulk composition. The best quality fit in terms of minimal $S^{2}=0.83$ corresponds to a chemical composition of the core made of a mixture of C-O of mass fraction 60/40 as can be seen in Fig. 1 of Giammichele et al. (2014, in press). Cores composed of pure carbon and oxygen can be unambiguously ruled out from the poor matches of the observed frequency spectrum. If we consider the newest spectroscopic determination of $T_{\text {eff }}$ and $\log g$ for R548, the mixed C-O core is the solution that agrees best with spectroscopic
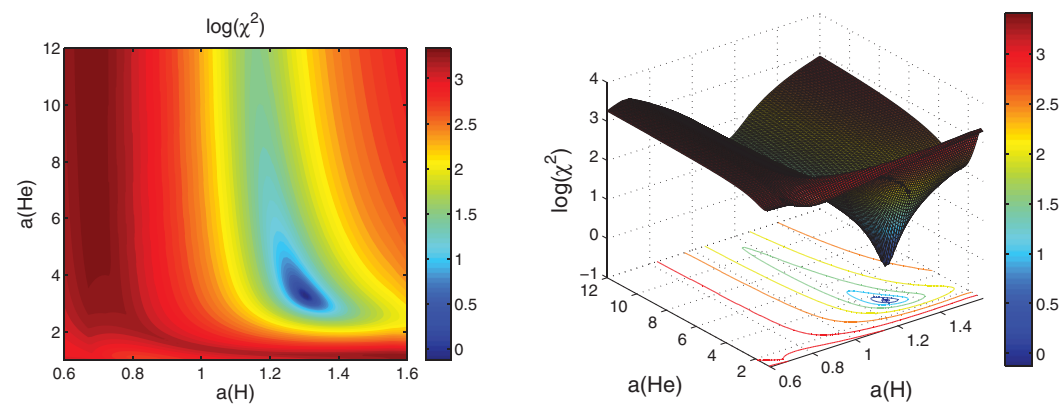

Figure 2. Contour and 3-D map of the merit function $S^{2}$ as a function of the values of the chemical profile steepness at the interface between the $\mathrm{H} / \mathrm{He}$ and $\mathrm{He} / \mathrm{C}-\mathrm{O}$ layers, for the optimal effective temperature and surface gravity. 


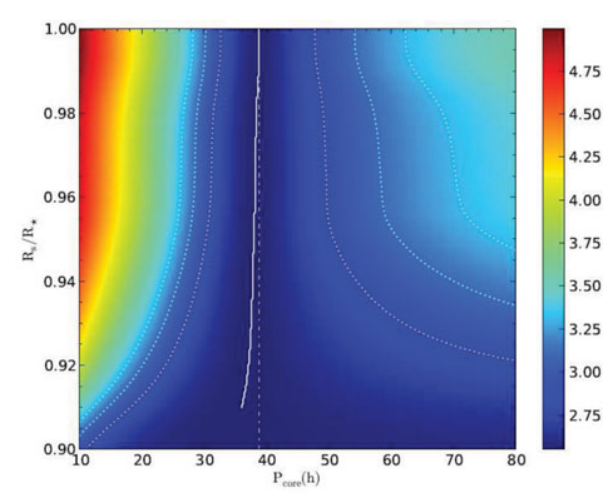

Figure 3. Contour map showing the behaviour of the normalized merit function $S^{2}$ in terms of the radius and in terms of the rotation period of the inner region for solid body rotation in a two-zone approach defined by Charpinet et al. (2009b). The solution is illustrated by the nearly vertical white curve and the dotted white curves depict the 1,2 and $3 \sigma$ contours. In comparison, the vertical dot-dashed white curve gives the exact solution for solid-body rotation.

parameters while displaying the best (lowest) value of the merit function $S^{2}$ of the seismic solution. This contributes in giving credibility to the proposed method.

It must be pointed out that it is the imposed smoothness of the core profile (assumed homogeneously mixed) that has allowed us to carry out successfully our asteroseismic exercise. Indeed, through numerous tests, we found that detailed post-AGB models contain too much noise in the core for pursuing quantitative seismology. For example, we implemented in some of our test models the detailed core chemical profiles computed by Salaris et al. (2010). Even if the grid of optimized parameters is showing the same global geometry, the surface turns out to be too noisy to identify a global minimum. We believe that current post-AGB models leave core structures that are most probably not physical, rendering the seismic exercise difficult if not impossible.

\subsection{Rotation profile structure}

Based on the rotational splitting (Giammichele et al. 2013a) shown in four out of the five modes listed in Table 1 and on the optimal model of R548, we determined whether or not its internal rotation profile corresponds to solid-body rotation, as seen in Figure 3. Unfortunately, evolution has pushed the $g$-modes to the outer part of the star because of the increase of degeneracy along the cooling track. Unlike the case of GW Vir stars (see Charpinet et al. 2009b), rotation kernels have large amplitudes in the outermost layers only. Therefore, we can only probe about $10 \%$ of the radius, and less than $1 \%$ of the total mass. But within the outer $10 \%$ radius, we can assert that R548 is rotating as a solid body. The relatively low period of $39 \mathrm{~h}$ is consistent with the suggestion of Charpinet et al. (2009b) to the effect that isolated white dwarfs must have lost essentially all of their angular momentum during evolutionary phases prior to the white dwarf stage.

\section{References}

Charpinet, S., Fontaine, G., \& Brassard, P. 2009a, A\&A, 493, 595

Charpinet, S., Fontaine, G., \& Brassard, P. 2009b, Nature, 461, 501

Giammichele, N., Fontaine, G., \& Brassard, P. 2013a, European Physical Journal Web of Conferences, 43, 5007

Giammichele, N., Fontaine, G., \& Brassard, P. 2013b, ASP-CS, 469, 49

Salaris, M., Cassisi, S., Pietrinferni, A., Kowalski, P. M., \& Isern, J. 2010, ApJ, 716, 1241 